\title{
Editorial corner - a personal view Polyvinylidene fluoride: a versatile polymer for biomedical, electronic, energy and environmental applications
}

\author{
S. C. Tjong ${ }^{*}$ \\ Department of Physics, City University of Hong Kong, Kowloon, Hong Kong
}

Polyvinylidene fluoride (PVDF) has received considerable attention recently due to its superior chemical resistance, thermal stability and excellent mechanical strength. PVDF exhibits four crystalline forms including $\alpha, \beta, \gamma$ and $\delta$ depending on the crystallization and processing conditions. Processing factors are known to affect the properties of polymers greatly (https:// doi.org/10.1016/S0032-3861(97)00218-8 and https:// doi.org/10.1016/S0032-3861(96)01082-8). Among these phases, polar $\beta$-PVDF exhibits exceptionally good pyroelectric, piezoelectric and dielectric properties. It can be induced by mechanical stretching, electrical poling, electrospinning or nanomaterial additions (https://doi.org/10.1016/j.carbon.2010.12.062). PVDF membranes have a high binding capacity for proteins and are commonly used for Western blotting. This method involves the transfer of protein patterns from gel electrophoresis onto a PVDF membrane. Piezoelectric PVDF films have also been widely considered in biosensor (https://doi.org/10.3144/expresspolymlett.2018.12), tissue engineering and regeneration applications (https://doi.org/10.1016/j. colsurfb.2015.08.043). PVDF with high dielectric permittivity and low dielectric loss is an attractive material for electrical charge storage and microelectronic devices. This can be achieved by adding barium titanate (BT) nanoparticles, BT/graphene and BT/SiC hybrids to PVDF (https://doi.org/10.3144/expresspolymlett.2011.51; https://doi.org/10.1016/j.elstat.2013.08.004 and https://doi.org/10.1016/j.compositesa.2015.04.002).

\footnotetext{
*Corresponding author, e-mail: APTJONG@,cityu.edu.hk (c) BME-PT
}

In recent years, climate change, population growth, urbanization and industrialization have led to environmental pollution and water scarcity worldwide. Replacing fossil fuels with alternative energy sources would reduce air pollution markedly. PVDF and copolymers have been adopted for clean energy applications such as the membranes of fuel cells and the separators of lithium ion batteries (https://doi.org/10. 1016/j.progpolymsci.2013.07.008). In the latter case, electrospun PVDF and its copolymer membranes exhibit good electrochemical properties, including high ionic conductivity, wide electrochemical stability window and good cycling performance (https://doi. org/10.1021/cm060223+). Modern wastewater treatment plants require advanced porous membranes with enhanced water flux permeation and antifouling properties. So hydrophobic PVDF membranes are largely used in microfiltration and ultrafiltration processes. However, they suffer from protein and bacterial fouling. Thus surface modification and inorganic nanomaterials have been employed to improve hydrophilicity and antifouling resistance of PVDF (https://doi. org/10.1016/j.memsci.2011.03.014).

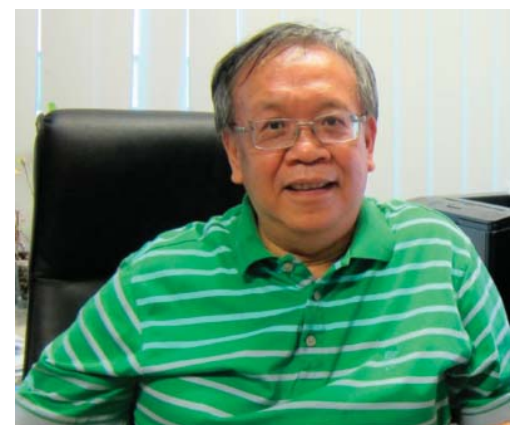

Prof. Dr. Sie Chin Tjong

Member of the International Advisory Board 